\title{
THE ZEROS OF A CERTAIN CLASS OF INDEFINITE INTEGRALS
}

ARTHUR E. LIVINGSTON ${ }^{1}$

1. Introduction. The zeros modulo 1 of the familiar function

$$
\text { si }(\pi x)=\int_{x}^{\infty} t^{-1} \sin \pi t d t, \quad x>0,
$$

can be shown to have $1 / 2$ as their only limit point. This result leads quite naturally to an investigation of the limit points of the zeros modulo 1 of the function $F(x)=\int_{x}^{\infty} f(t) g(t) d t$, where $f(t)$ and $g(t)$ are functions defined for $t \geqq 0$ and subject to the following conditions:

H1. $f(t) \geqq 0$ and is Lebesgue integrable on $0 \leqq t \leqq 1$, and $f(t+n)$ $=(-1)^{n} f(t)$ for $n=1,2, \cdots$.

H2. $f(t) \not \equiv 0$ on any subinterval of $0 \leqq t<1$.

H3. $g(t)>0$, for $t \geqq 0$, and $f(t) g(t)$ is Lebesgue integrable on $0 \leqq t \leqq 1$.

H4. $g(t)$ is monotone decreasing to zero for $0 \leqq t<\infty$.

H5. $g(t)$ has a point of decrease in each interval $n_{k}<t<n_{k}+1$, where $\left(n_{k}\right)_{k=0}^{\infty}$ is an infinite set of distinct non-negative integers:

H6. $g(n+1) / g(n) \rightarrow 1$ as $n \rightarrow \infty, n=0,1, \cdots$.

Easily deducible properties of the function $F(x)$ are summarized in

TheOREM 1.1. Conditions H1-H5 imply that

(a) $F(x)$ is defined for $x \geqq 0$ and is not identically constant on any subinterval of $0 \leqq x<\infty$;

(b) the relative maxima (minima) of $F(x)$ occur at $x=2 n(x=2 n+1)$, $n=0,1, \cdots$;

(c) $F(x)$ has precisely one zero, call it $z_{n}$, in each interval $n<x<n+1$, $n=0,1, \cdots ;$ and

(d) $0 \leqq(-1)^{n} F(n) / g(n) \leqq \int_{0}^{1} f(t) d t, n=0,1, \cdots$.

If $N$ is any infinite set of non-negative integers and $\alpha$ is any number such that $0 \leqq \alpha \leqq 1$, let propositions $\mathrm{A}(\alpha, N)$ and $\mathrm{B}(\alpha, N)$ be defined as follows:

$$
\mathrm{A}(\alpha, N): \quad(-1)^{n} F(n) / g(n) \rightarrow \int_{0}^{\alpha} f(t) d t \text { as } n \rightarrow \infty, \quad n \in N .
$$

Presented to the Society, February 28, 1953; received by the editors March 12, 1953 and, in revised form, July 13, 1953.

1 The author is a National Science Foundation Fellow. 


$$
\mathrm{B}(\alpha, N): z_{n}-n \rightarrow \alpha \text { as } n \rightarrow \infty, \quad n \in N,
$$

where $z_{n}$ is defined in Theorem 1.1(c). It will be shown that conditions H1-H6 imply that $\mathrm{A}(\alpha, N)$ is true if and only if $\mathrm{B}(\alpha, N)$ is true (Theorem 2.1).

Let $C$ be defined by the conditions

$$
0<C<1 \text { and } 2 \int_{0}^{C} f(t) d t=\int_{0}^{1} f(t) d t .
$$

(Note that the condition $\mathrm{H} 2$ implies that $C$ is uniquely determined by these conditions.) If $g(t)$ satisfies the additional condition

H7. $g(t)-g(t+1)$ is monotone decreasing for $0 \leqq t<\infty$, then it will be shown that the conditions $\mathrm{H} 1-\mathrm{H} 5$ and $\mathrm{H} 7$ are sufficient in order that the zeros modulo 1 of $F(x)$ lie to the right of $C$ (Theorem 3.1) and that conditions $\mathrm{H} 1-\mathrm{H} 7$ are sufficient to show that these zeros modulo 1 have $C$ as their only limit point (Theorem 3.3). An example is given to show that the condition $\mathrm{H} 7$ is necessary in order that these last two results be true in general.

2. This section is devoted to a proof of

THEOREM 2.1. The conditions H1-H6 of $\$ 1$ imply that the proposition $\mathrm{A}(\alpha, N)$ is true if and only if $\mathrm{B}(\alpha, N)$ is true.

PROOF: If $z_{n}-n \geqq \alpha$, then

$$
\begin{aligned}
0 \leqq \frac{g\left(z_{n}\right)}{g(n)} \int_{\alpha}^{z_{n}-n} f(t) d t & \leqq \frac{(-1)^{n}}{g(n)} \int_{n+\alpha}^{z_{n}} f(t) g(t) d t \\
& \leqq \frac{g(n+\alpha)}{g(n)} \int_{\alpha}^{z_{n}-n} f(t) d t,
\end{aligned}
$$

while for $z_{n}-n<\alpha$,

$$
\begin{aligned}
0 & \leqq-\frac{g(n+\alpha)}{g(n)} \int_{\alpha}^{z_{n}-n} f(t) d t \leqq \frac{(-1)^{n+1}}{g(n)} \int_{n+\alpha}^{z_{n}} f(t) g(t) d t \\
& \leqq-\frac{g\left(z_{n}\right)}{g(n)} \int_{\alpha}^{z_{n}-n} f(t) d t .
\end{aligned}
$$

Since H4 and H6 imply that $g(n+y) / g(n) \rightarrow 1$ uniformly for $|y| \leqq 1$, it follows that, as $n \rightarrow \infty$,

$$
\int_{\alpha}^{z_{n-n}} f(t) d t \rightarrow 0 \text { if and only if } \frac{(-1)^{n}}{g(n)} \int_{n+\alpha}^{z_{n}} f(t) g(t) d t \rightarrow 0 .
$$

Furthermore, conditions $\mathrm{H} 1, \mathrm{H} 4$, and $\mathrm{H} 6$ imply that 


$$
\frac{(-1)^{n}}{g(n)} \int_{n}^{n+\alpha} f(t) g(t) d t \rightarrow \int_{0}^{\alpha} f(t) d t
$$

as $n \rightarrow \infty$.

The proof of the theorem is now completed by employing (2.1) and (2.2) in the identity

$$
\begin{aligned}
0=\frac{(-1)^{n}}{g(n)} \int_{z_{n}}^{\infty} f(t) g(t) d t= & (-1)^{n} F(n) / g(n)-\frac{(-1)^{n}}{g(n)} \int_{n}^{n+\alpha} f(t) g(t) d t \\
& -\frac{(-1)^{n}}{g(n)} \int_{n+\alpha}^{z_{n}} f(t) g(t) d t .
\end{aligned}
$$

3. The first result to be proved in this section is

Theorem 3.1. If $z_{n}$ is defined as in Theorem 1.1(c) and $C$ is defined by (1.1), then the conditions $\mathrm{H} 1-\mathrm{H} 5$ and $\mathrm{H} 7$ imply that $z_{n}-n \geqq C$, $n=0,1, \cdots$.

Proof. By Theorem 1.1(b), it is sufficient to show that $(-1)^{n} F(n+C) \geqq 0$ for $n=0,1, \cdots$.

$$
\begin{aligned}
(-1)^{n} F(n+C) & =(-1)^{n} \int_{n+C}^{\infty} f(t) g(t) d t \\
& =(-1)^{n} \sum_{k=n}^{\infty} \int_{k+C}^{k+1+C} f(t) g(t) d t \\
& =(-1)^{n} \sum_{k=n}^{\infty}(-1)^{k} \int_{C}^{1+c} f(t) g(t+k) d t \\
& =\int_{C}^{1+C} f(t)\left\{\sum_{k=n}^{\infty}(-1)^{n+k} g(t+k)\right\} d t,
\end{aligned}
$$

where the interchange in the order of summation and integration is justified by Lebesgue's principle of dominated convergence.

Let

$$
g_{n}(t)=\sum_{k=n}^{\infty}(-1)^{n+k} g(t+k) .
$$

Then $g_{n}(t)>0$ and, by H7, is monotone decreasing in $t$ for each $n$. Therefore,

and

$$
\int_{C}^{1} f(t) g_{n}(t) d t \geqq g_{n}(1) \int_{C}^{1} f(t) d t=g_{n}(1) \int_{0}^{C} f(t) d t,
$$




$$
\int_{1}^{1+C}-f(t) g_{n}(t) d t \leqq g_{n}(1) \int_{0}^{c} f(t) d t
$$

so that

$$
\int_{C}^{1} f(t) g_{n}(t) d t \geqq \int_{1}^{1+C}-f(t) g_{n}(t) d t,
$$

which, because of (3.1) and (3.2), completes the proof.

TheOREM 3.2. The conditions $\mathrm{H} 1-\mathrm{H} 7$ imply that

$$
(-1)^{n} F(n) / g(n) \rightarrow \int_{0}^{c} f(t) d t
$$

as $n \rightarrow \infty$, where $C$ is defined by (1.1).

Proof. The formula

$$
(-1)^{n} F(n)=\int_{0}^{1} f(t) g_{n}(t) d t
$$

where $g_{n}(t)$ is given by (3.2), is obtained and justified in the same way as was (3.1). Since $g_{n}(t)$ is decreasing in $t$ for each $n$, (3.3) gives

$$
g_{n}(1) \int_{0}^{1} f(t) d t \leqq(-1)^{n} F(n) \leqq g_{n}(0) \int_{0}^{1} f(t) d t .
$$

Recalling that $g(x)-g(x+1)$ is nonincreasing, one sees that

$$
\begin{aligned}
2 g_{n}(1) & =2 \sum_{k=0}^{\infty}[g(n+1+2 k)-g(n+2+2 k)] \\
& \geqq \int_{0}^{\infty} 2[g(n+1+2 t)-g(n+2+2 t)] d t \\
& =\int_{n+1}^{\infty}[g(x)-g(x+1)] d x=\lim _{R \rightarrow \infty} \int_{n+1}^{R}[g(x)-g(x+1)] d x \\
& =\int_{n+1}^{n+2} g(x) d x-\lim _{R \rightarrow \infty} \int_{R}^{1+R} g(x) d x=\int_{n+1}^{n+2} g(x) d x \\
& \geqq g(n+2),
\end{aligned}
$$

where the last integral involving $R$ vanishes because of H4. In a similar way, it is found that $2 g_{n}(0) \leqq g(n-1)$ for $n>1$. Employing these inequalities in (3.4), taking the limit as $n \rightarrow \infty$, and recalling H6 completes the proof.

Combining Theorem 3.2 with Theorem 2.1 gives 
TheOREM 3.3. The conditions $\mathrm{H} 1-\mathrm{H} 7$ imply that $z_{n}-n \rightarrow C$ as $n \rightarrow \infty$, $n=0,1, \cdots, z_{n}$ as defined in Theorem 1.1(c).

If $f(t)=\sin \pi t$ and $g(t)=t^{-1}$, then $f(t)$ and $g(t)$ satisfy conditions H1-H7 (since $g(t)$ is convex and monotone decreasing, it automatically satisfies $\mathrm{H} 7)$, and the $F(x)$ obtained is $\mathrm{si}(\pi x)$. By Theorem 3.3 , the zeros modulo 1 of $\operatorname{si}(\pi x)$ tend to $1 / 2$. (Actually, more than this is known, for Harry Pollard has shown that the sequence $\left(z_{n}-n\right)_{0}^{\infty}$ is monotone decreasing. Substantially his proof of this result is given in A. E. Livingston, Some Hausdorff means which exhibit the Gibbs' phenomenon, Pacific Journal of Mathematics vol. 3 (1953) pp. 407-415.)

4. Concluding remarks. There will now be exhibited a function $g_{\epsilon}(t)$ which, for suitable choice of $\epsilon$, will serve to illustrate that the condition $\mathrm{H} 7$ is not necessary for the validity of Theorems 3.1 and 3.2 in certain special instances and, yet, can not be relaxed if one wishes either of these two theorems to hold in general.

ExAmple. Let $0<\epsilon<1$ and define $g_{\epsilon}(t)= \begin{cases}1 & \text { for } 0 \leqq t<\epsilon, \\ (n+1)^{-1} & \text { for } 2 n-2+\epsilon \leqq t<2 n+\epsilon, \quad n=1,2, \cdots .\end{cases}$

It is a simple matter to verify that conditions $\mathrm{H} 3-\mathrm{H} 6$ are satisfied by $g_{\epsilon}(t)$ for each $\epsilon$ and that $g_{\epsilon}(t)-g_{\epsilon}(t+1)$ does not satisfy H7. One easily finds that

$$
F_{\epsilon}(2 n) / g_{\epsilon}(2 n)=\int_{0}^{\epsilon} f(t) d t, \quad-F_{\epsilon}(2 n+1) / g_{\epsilon}(2 n+1)=\int_{\epsilon}^{1} f(t) d t,
$$

so that the conclusion of Theorem 3.2 is obtained if $\epsilon=C, C$ as defined in (1.1). Further, $z_{2 n}=2 n+\epsilon$, and $z_{2 n+1}<C$ if $\epsilon>C, z_{2 n+1} \geqq C$ if $\epsilon \leqq C$.

This example further illustrates that the zeros modulo 1 of $F(x)$ can have more than one limit point.

The condition H6 is a rather stringent requirement. However, the example $f(t)=\pi \sin \pi t, g(t)=e^{-\pi t}$ shows that it can not be relaxed, for, in this case, $z_{n}-n=3 / 4$, while

$$
(-1)^{n} F(n) / g(n)=1 / 2=\int_{0}^{1 / 3} f(t) d t .
$$

Thus, Theorems 2.1, 3.2, and 3.3 are not true in general if the hypothesis $\mathrm{H} 6$ is removed.

The Institute for Advanced Study 